\title{
Erratum to: Homology stability for outer automorphism groups of free groups
}

\author{
Allen Hatcher \\ KAREN VOGTMANN \\ NATHALIE WAHL
}

\begin{abstract}
We correct the proof of Theorem 5 of the paper Homology stability for outer automorphism groups of free groups, by the first two authors.
\end{abstract}

20F65; 20F28, 57M07

In Hatcher-Vogtmann [2] a proof was presented that the homology of certain groups $\Gamma_{n, s}$ is independent of both $n$ and $s$ for $n$ sufficiently large. The groups $\Gamma_{n, s}$ include $\operatorname{Aut}\left(F_{n}\right)(s=1)$ and $\operatorname{Out}\left(F_{n}\right)(s=0)$. In August of 2005 Nathalie Wahl discovered an error in the proof, and the purpose of this note is to fix that error.

We assume the reader is familiar with [2], whose notation and conventions we will use here without further comment. The error occurs in the first part of the proof of Theorem 5, showing that the map $\beta_{*}: H_{i}\left(\Gamma_{n, s+2}\right) \rightarrow H_{i}\left(\Gamma_{n+1, s}\right)$ is injective for $n \gg i$ and $s \geq 1$. The argument used a diagram chase in the following diagram:

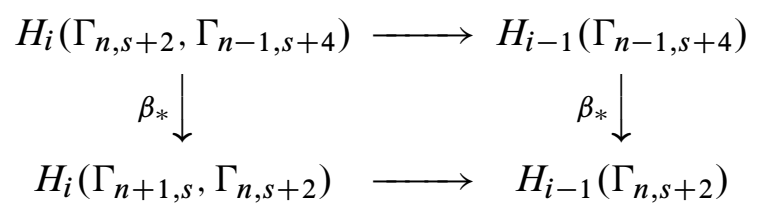

It was asserted that the top horizontal and right vertical arrows were successive maps in the long exact sequence of the pair $\left(\Gamma_{n, s+2}, \Gamma_{n-1, s+4}\right)$, and hence their composition was the zero map, but in fact the group $\Gamma_{n, s+2}$ in the lower right corner of the diagram is a different subgroup of $\Gamma_{n+1, s}$ from the $\Gamma_{n, s+2}$ in the upper left corner, so that $\beta_{*}$ is not induced by the inclusion map of the pair. It is in fact true that the composition is the zero map for $n$ sufficiently large, but a proof seems to require the results proved in this correction.

We correct the problem by giving a completely new proof of stability with respect to $s$ for $s \geq 1$, complementing the earlier proof of stability with respect to $n$. The new proof entirely avoids the diagram displayed above and instead focuses on the map $\mu: \Gamma_{n, s} \rightarrow \Gamma_{n, s+1}$. In the range where $\alpha_{*}$ is an isomorphism, the relation $\alpha=\beta \mu^{2}$ 
derived in section 2 of [2] shows that $\beta_{*}$ is an isomorphism if and only if $\mu_{*}$ is an isomorphism. Note that $\mu_{*}$ is always injective since it has a left inverse obtained by gluing a disk to one of the new boundary components, so we only need to prove surjectivity of $\mu_{*}$ in a stable range. The commutative diagram

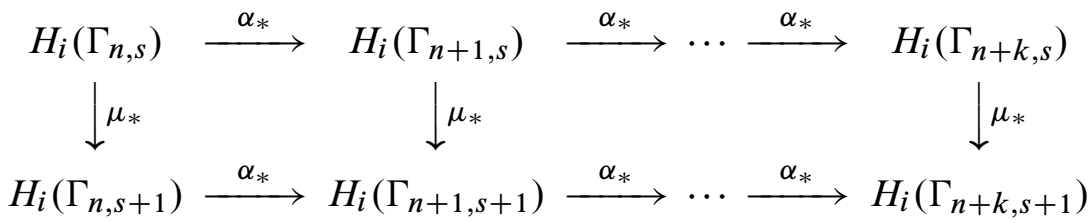

shows that if $\mu_{*}$ is an isomorphism for $n>>i$ then it will be an isomorphism in the same range that $\alpha$ is an isomorphism, namely $n \geq 2 i+2$. It is thus not necessary to keep track of the precise stable range in the arguments given in this correction.

For $s \geq 2$ let $\eta: \Gamma_{n, s} \rightarrow \Gamma_{n+1, s-1}$ be induced by gluing a copy of the 3-punctured sphere $M_{0,3}$ to the first and last boundary components of $M_{n, s}$. The stabilization $\alpha$ is the composition $\eta \mu: \Gamma_{n, s} \rightarrow \Gamma_{n+1, s}$, and now we want to consider the opposite composition $\mu \eta: \Gamma_{n, s} \rightarrow \Gamma_{n+1, s}$. We will show that $\mu \eta$ is an isomorphism on $H_{i}$ for $n>>i$, and hence $\mu_{*}: H_{i}\left(\Gamma_{n, s-1}\right) \rightarrow H_{i}\left(\Gamma_{n, s}\right)$ is surjective for $n>>i$ and $s \geq 2$. Since $\alpha$ is a homology isomorphism for $n>>i$, and, as we will see, $\alpha$ commutes with $\mu \eta$, it will suffice to show that $\mu \eta$ is a homology isomorphism after passing to the direct limit under stabilization by $\alpha$. This turns out to be much easier than showing $\mu \eta$ is a homology isomorphism before passing to the limit.

To prove that $\mu \eta$ is a homology isomorphism in the limit we use a new simplicial complex $Z_{n, s}$, defined for $s \geq 2$. To simplify the notation we will omit the subscript $s$ since it will be fixed throughout the proof, so we write $Z_{n, s}$ as $Z_{n}$ and $M_{n, s}$ as $M_{n}$. A vertex of $Z_{n}$ is an equivalence class of pairs $(S, a)$ where $S$ is a non-separating sphere in $M_{n}$ and $a$ is an embedded arc in $M_{n}$ joining the first and last boundary spheres $\partial_{0}$ and $\partial_{s-1}$ and intersecting $S$ transversely in one point; we refer to $a$ as a dual arc for $S$. The equivalence relation on such pairs $(S, a)$ is given by isotopy of $S \cup a$ keeping the endpoints of $a$ in $\partial M_{n}$. A set of $k+1$ vertices $\left(S_{0}, a_{0}\right), \cdots,\left(S_{k}, a_{k}\right)$ forms a $k$-simplex if $S_{i} \cup a_{i}$ is disjoint from $S_{j} \cup a_{j}$ for $i \neq j$ and the spheres $S_{i}$ form a coconnected system (see Figure 1). This implies that no two $S_{i}$ 's or $a_{i}$ 's are isotopic. Note that arcs can always be made disjoint by general position, so the disjointness condition really only involves intersections between different spheres and between spheres and arcs.

We note that there is an equivalent way of viewing a simplex of $Z_{n}$ in terms of enveloping sphere-pairs. For a simplex $\left\{\left(S_{0}, a_{0}\right), \cdots,\left(S_{k}, a_{k}\right)\right\}$, take two parallel copies of each $S_{i}$, one on either side of $S_{i}$, and join each of these new spheres 


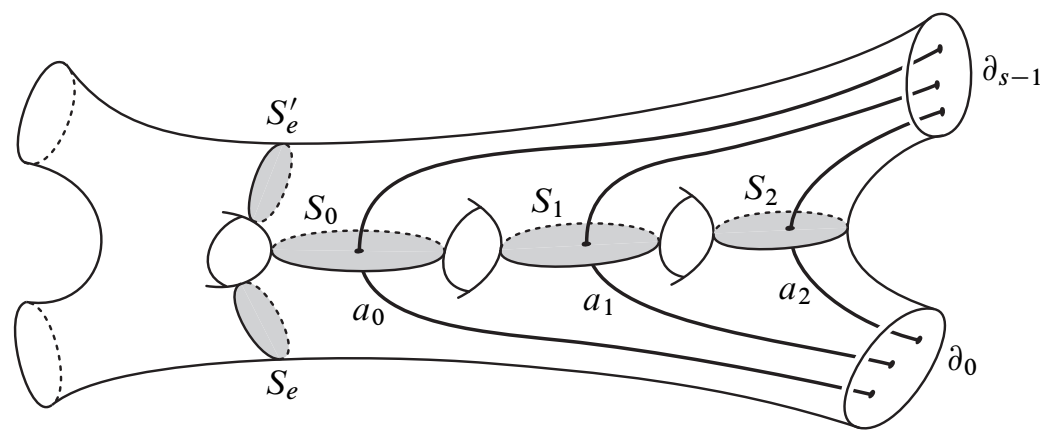

Figure 1: A 2-simplex in $Z_{n, s}$

to a sphere parallel to either $\partial_{0}$ or $\partial_{s-1}$ by a tube following the half of $a_{i}$ on the appropriate side of $S_{i}$. This produces a pair of spheres $S_{e}, S_{e}^{\prime}$ separating $M_{n}$ into two components, one of which contains the spheres $S_{i}$. The only boundary spheres contained in this component are $\partial_{0}$ and $\partial_{s-1}$, and splitting this component along the spheres $S_{i}$ produces two simply-connected pieces, one bounded by $S_{e}, \partial_{0}$, and the $S_{i}$ 's, the other bounded by $S_{e}^{\prime}, \partial_{s-1}$, and the $S_{i}$ 's. Conversely, given a coconnected system $S_{0}, \cdots, S_{k}$ and two spheres $S_{e}, S_{e}^{\prime}$ with the properties just listed, then there are dual $\operatorname{arcs} a_{i}$ in the split-off submanifold such that $\left\{\left(S_{0}, a_{0}\right), \cdots,\left(S_{k}, a_{k}\right)\right\}$ is a simplex of $Z_{n}$, and these $a_{i}$ 's are unique up to isotopy.

We wish to describe now an inclusion $Z_{n} \hookrightarrow Z_{n+1}$. This will be induced by an inclusion $M_{n} \hookrightarrow M_{n+1}$. We have already used one such inclusion in the definition of the stabilization $\alpha: \Gamma_{n, s} \rightarrow \Gamma_{n+1, s}$ when we regarded $M_{n+1}$ as being obtained from $M_{n}$ by attaching $M_{1,2}$ to $\partial_{0}$ along one boundary sphere of $M_{1,2}$. However, an alternative approach will make things a little clearer when dealing with the complexes $Z_{n}$. Here we build $M_{n+1}$ from $M_{n}$ by attaching $M_{1,1}$, identifying a disk in $\partial M_{1,1}$ with a disk in $\partial_{0}$ (see Figure 2). The first inclusion $M_{n} \hookrightarrow M_{n+1}$ is then recovered by attaching a product $S^{2} \times I$ to the new $\partial_{0}$. Since attaching this product does not affect isotopy classes of diffeomorphisms modulo Dehn twists, the new inclusion $M_{n} \hookrightarrow M_{n+1}$ gives the same $\alpha$ as the old one.

The new inclusion $M_{n} \hookrightarrow M_{n+1}$ induces a map $Z_{n} \rightarrow Z_{n+1}$ since we may assume simplices of $Z_{n}$ are represented using pairs $\left(S_{i}, a_{i}\right)$ whose arcs $a_{i}$ are disjoint from the disk in $\partial_{0}$ where $M_{1,1}$ is attached. This map $Z_{n} \rightarrow Z_{n+1}$ is injective by general properties of sphere systems (uniqueness of normal forms Hatcher [1]). Let $Z_{\infty}$ denote the direct limit of the complexes $Z_{n}$ under these inclusions $Z_{n} \hookrightarrow Z_{n+1}$.

Lemma $Z_{\infty}$ is contractible. 


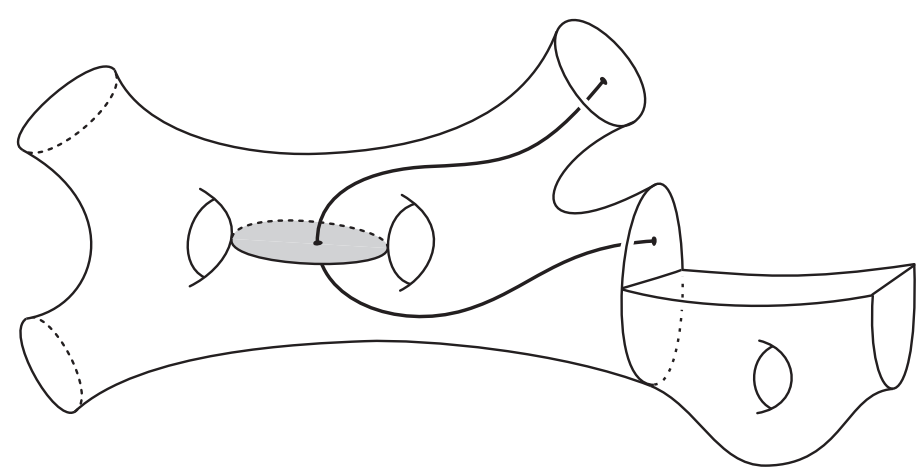

Figure 2: Stabilization by $\alpha$ and the inclusion $Z_{n} \hookrightarrow Z_{n+1}$

Proof Given a map $g$ : $S^{k} \rightarrow Z_{\infty}$, we wish to extend this to a map $D^{k+1} \rightarrow Z_{\infty}$. We may assume $g$ is simplicial with respect to some triangulation of $S^{k}$. This triangulation has finitely many simplices, so the image of $g$ lies in $Z_{n}$ for some $n$. Let $M_{n} \subset M_{n+1} \subset \cdots \subset M_{n+k+1}$ be the alternate inclusions described above inducing $\alpha$, and choose a non-separating sphere $T_{i}$ in each $M_{n+i+1}-M_{n+i}$.

Triangulate $D^{k+1}$ by coning off the triangulation of $S^{k}$ to the centerpoint of $D^{k+1}$, a new vertex $v$. Define $g(v)$ to be $\left(T_{0}, b_{v}\right)$ where $b_{v}$ is any arc in $M_{n+1}$ dual to $T_{0}$. Next, we extend $g$ over each interior edge $e$ of $D^{k+1}$ in the following way. The endpoints of $e$ map to $\left(T_{0}, b_{v}\right)$ and to another vertex $\left(S_{0}, a_{0}\right)$. Let $g$ send the midpoint of $e$ to $\left(T_{1}, b_{e}\right)$, where $b_{e}$ is an arc in $M_{n+2}$ which is in the complement of the coconnected system $\left\{S_{0}, T_{0}\right\}$. Then $\left\{\left(S_{0}, a_{0}\right),\left(T_{1}, b_{e}\right)\right\}$ and $\left\{\left(T_{1}, b_{e}\right),\left(T_{0}, b_{v}\right)\right\}$ are edges of $Z_{n+2}$ so $g$ extends over $e$ by mapping its two halves to these two edges.

The extension of $g$ over simplices of $D^{k+1}$ of higher dimension proceeds in a similar fashion, by induction on the dimension of the simplices. Each $i$-simplex $\sigma$ of $D^{k+1}$ not contained in $S^{k}$ is the cone to $v$ of an $(i-1)$-simplex in $S^{k}$. The map $g$ sends this $(i-1)$-simplex to a possibly degenerate simplex $\left\{\left(S_{0}, a_{0}\right), \cdots,\left(S_{i-1}, a_{i-1}\right)\right\}$ in $Z_{n}$. The rest of the boundary of $\sigma$ is sent by induction to a subcomplex with additional vertices $\left(T_{j}, b_{\tau}\right)$ for $0 \leq j<i$, where $\tau$ ranges over the faces of $\sigma$ not in $S^{k}$. We send the barycenter of $\sigma$ to $\left(T_{i}, b_{\sigma}\right)$ where $b_{\sigma}$ is chosen in $M_{n+i+1}$ and in the complement of the coconnected system $\left\{S_{0}, \cdots, S_{i-1}, T_{0}, \cdots, T_{i-1}\right\}$. We can then extend $g$ over $\sigma$ by coning off to its barycenter. This gives the induction step, and at the end of the induction we have extended $g$ over $D^{k+1}$. Since $g$ and $k$ were arbitrary, this shows $Z_{\infty}$ is contractible.

The natural action of $\Gamma_{n}$ on $Z_{n}$ is transitive on simplices of each dimension, since splitting $M_{n}$ along the $k+1$ spheres of a $k$-simplex produces the manifold $M_{n-k-1}$ 
with $2 k+2$ new punctures, each joined to $\partial_{0}$ or $\partial_{s-1}$ by an arc, and any two such configurations are diffeomorphic. The action of $\Gamma_{n}$ on $Z_{n}$ is compatible with the stabilization $\alpha$, in the sense that for each $g \in \Gamma_{n}$ the following diagram commutes:

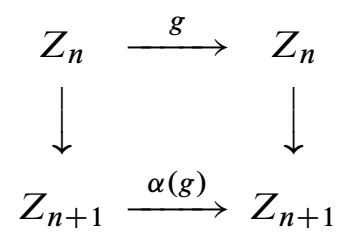

Thus the direct limit group $\Gamma_{\infty}$ acts on $Z_{\infty}$. This action is also transitive on $k-$ simplices for each $k$.

For the action of $\Gamma_{\infty}$ on $Z_{\infty}$ the stabilizer of a simplex includes group elements that permute the vertices of the simplex, so to avoid this we consider $U_{\infty}=\Delta\left(Z_{\infty}\right)$, the complex whose $k$-simplices are all the simplicial maps from the standard $k$-simplex to $Z_{\infty}$. Note that $U_{\infty}$ is the direct limit of the complexes $U_{n}=\Delta\left(Z_{n}\right)$. The homology of $U_{\infty}$ is trivial since $Z_{\infty}$ has trivial homology. The action of $\Gamma_{\infty}$ on $Z_{\infty}$ induces an action on $U_{\infty}$. The quotient $U_{\infty} / \Gamma_{\infty}$ is contractible since it is the direct limit of the quotients $U_{n} / \Gamma_{n}$ and these quotients are combinatorially the same as the quotients $W_{n} / \Gamma_{n}$ in the proof of Theorem 4 of [2], which were $(n-2)$-connected.

The stabilizer of a vertex for the action of $\Gamma_{n}$ on $U_{n}$ is a copy $\Gamma_{n-1}^{\prime}$ of $\Gamma_{n-1}$ in $\Gamma_{n}$, and the inclusion of this stabilizer is the map $\mu \eta$. The map $\mu \eta$ is induced by gluing a four-punctured 3-sphere to $M_{n}$ by attaching two of its boundary spheres to $\partial_{0}$ and $\partial_{s-1}$. As in the case of $\alpha$, there is an alternative description of $\mu \eta$ as being induced by gluing a twice-punctured 3-sphere to $M_{n}$ by attaching its boundary spheres to $\partial_{0}$ and $\partial_{s-1}$ along disks (see Figure 3 ).

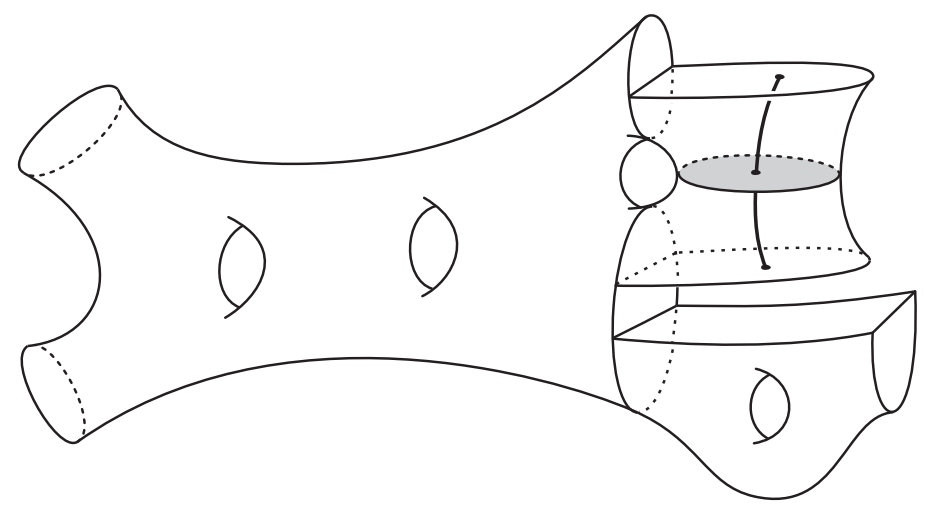

Figure 3: Compatibility of $\mu \eta$ with $\alpha$ 
This alternative description of $\mu \eta$ makes it clear that $\mu \eta$ commutes with $\alpha$, giving a commutative diagram:

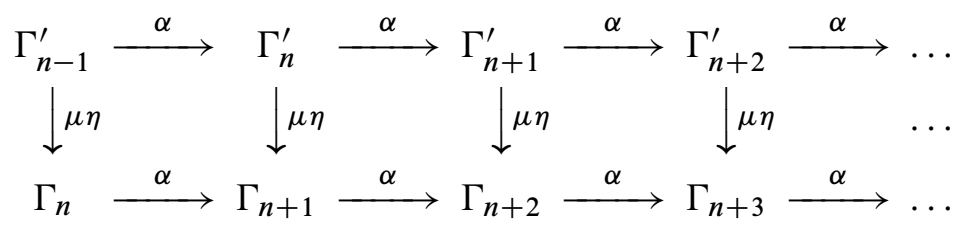

Thus in the limit action of $\Gamma_{\infty}$ on $U_{\infty}$ the inclusion of a vertex stabilizer is the direct limit map $\mu \eta: \Gamma_{\infty}^{\prime} \rightarrow \Gamma_{\infty}$. Similarly, for stabilizers of higher dimensional simplices the inclusions of stabilizers are iterates of $\mu \eta$.

We can now use the equivariant homology spectral sequence arising from the action of $\Gamma_{\infty}$ on $U_{\infty}$ to prove:

Theorem The map $(\mu \eta)_{*}: H_{i}\left(\Gamma_{\infty}^{\prime}\right) \rightarrow H_{i}\left(\Gamma_{\infty}\right)$ is an isomorphism for each $s \geq 2$.

As explained earlier, this implies:

Corollary The map $\mu_{*}: H_{i}\left(\Gamma_{n, s}\right) \rightarrow H_{i}\left(\Gamma_{n, s+1}\right)$ is an isomorphism when $n \geq 2 i+2$ and $s \geq 1$.

Proof of the theorem The proof proceeds by induction on $i$. The equivariant homology spectral sequence has

$$
E_{p, q}^{1}=\bigoplus_{\sigma_{p}} H_{q}\left(\operatorname{stab}\left(\sigma_{p}\right)\right) \Rightarrow \widetilde{H}_{p+q}\left(U_{\infty}\right)
$$

where $\left\{\sigma_{p}\right\}$ is a chosen set of orbit representatives for the $p$-simplices of $U_{\infty}$. The differential $d^{1}: E_{0, i}^{1} \rightarrow E_{-1, i}^{1}$ is the map $\mu \eta: \Gamma_{\infty}^{\prime} \rightarrow \Gamma_{\infty}$ we are interested in.

The $j^{\text {th }}$ row of the $E^{1}$ page of the spectral sequence is a chain complex computing the homology of the quotient $U_{\infty} / \Gamma_{\infty}$ with local coefficients in the system of groups $H_{j}\left(\operatorname{stab}\left(\sigma_{p}\right)\right)$. Each face of the boundary of $\sigma_{p}$ is equal to $h \sigma_{p-1}$ for some $\sigma_{p-1}$ and some element $h \in \Gamma_{\infty}$, and the corresponding term of the $d^{1}$ map is the map $h_{*}: H_{j}\left(\operatorname{stab}\left(\sigma_{p}\right)\right) \rightarrow H_{j}\left(\operatorname{stab}\left(\sigma_{p-1}\right)\right)$ induced by conjugation by $h$. For $j<i$ we may assume by induction that the vertical maps in the commutative diagram below are isomorphisms.

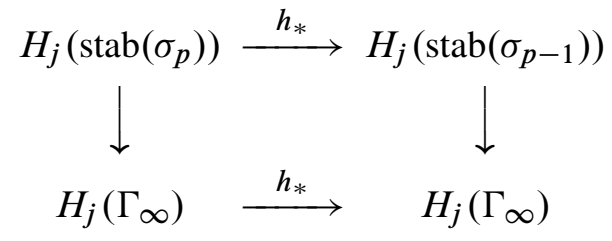

Algebraic 83 Geometric Topology, Volume 6 (2006) 
The lower $h_{*}$ in this diagram is the identity since it is induced by an inner automorphism of $\Gamma_{\infty}$. Thus the local coefficient system is trivial. (Here we are following a line of reasoning that can be found in Ivanov [3, Section 7.4].)

Since the quotient has trivial homology, this shows that the entire $E^{1}$ page below the $i^{\text {th }}$ row is zero. The spectral sequence converges to 0 since $U_{\infty}$ is contractible, and the only differential with a chance of killing $E_{-1, i}^{1}$ is $d^{1}=\mu \eta$, proving that this map must be onto.

To finish the induction we need to show that $\mu \eta$ is in fact an isomorphism on $H_{i}\left(\Gamma_{\infty}^{\prime}\right)$. Since $\mu \eta$ is surjective on $H_{i}\left(\Gamma_{\infty}^{\prime}\right)$, it is also surjective as a map $H_{i}\left(\Gamma_{n-1, s}^{\prime}\right) \rightarrow H_{i}\left(\Gamma_{n, s}\right)$ for large $n$ since $\alpha$ is an isomorphism on $H_{i}$ for large $n$. Therefore $\mu_{*}: H_{i}\left(\Gamma_{n, s-1}\right) \rightarrow$ $H_{i}\left(\Gamma_{n, s}\right)$ is surjective for large $n$, and hence an isomorphism. Since $\alpha=\eta \mu$ this implies that $\eta_{*}: H_{i}\left(\Gamma_{n, s}^{\prime}\right) \rightarrow H_{i}\left(\Gamma_{n+1, s-1}\right)$ is an isomorphism for large $n$, so that $\mu \eta$ is also an isomorphism on $H_{i}\left(\Gamma_{n, s}^{\prime}\right)$ for large $n$, hence on $H_{i}\left(\Gamma_{\infty}^{\prime}\right)$ as well.

\section{References}

[1] A Hatcher, Homological stability for automorphism groups of free groups, Comment. Math. Helv. 70 (1995) 39-62 MR1314940

[2] A Hatcher, K Vogtmann, Homology stability for outer automorphism groups of free groups, Algebr. Geom. Topol. 4 (2004) 1253-1272 MR2113904

[3] N V Ivanov, Complexes of curves and Teichmüller modular groups, Uspekhi Mat. Nauk 42 (1987) 49-91, 255 MR896878

AH, KV: Department of Mathematics, Cornell University, Ithaca, NY 14853, USA

NW: Department of Mathematics, University of Chicago, Chicago, IL 60637, USA

hatcher@math.cornell.edu, vogtmann@math.cornell.edu,

wahl@math.uchicago.edu

Received: 1 September 2005

Algebraic 83 Geometric Topology, Volume 6 (2006) 